\title{
Thermal mapping for detection of inhomogeneities in asphalt bridge decks
}

\author{
by H. Lorenzl ${ }^{*}$, M. Wistuba* and J. Grönniger*
}

* Braunschweig Pavement Engineering Centre, Technische Universität Braunschweig, Beethovenstraße 51b, 38106 Braunschweig, Germany, h.lorenzl@tu-bs.de

\begin{abstract}
Inhomogeneities in asphalt pavements such as blisters or loss of bonding between individual layers on bridge decks is an important issue as concerns quality of construction and durability of road pavements. To date, neither a reliable test procedure exists for quality assurance, nor a practical tool for analysis of damage. Hence, the development of a non destructive procedure is needed. Against this background, thermography is a well known method for analysis of energy flows in various fields of construction engineering. The article presents first results from the use of thermography for damage analysis on asphalt bridge decks and reflects important influencing factors to be taken into account for interpretation.
\end{abstract}

\section{Introduction}

\subsection{Damage on bridge decks}

Bridge constructions are exposed to weather and hence need a proper sealing to protect the supporting structure against water intrusion. The waterproofing is reached by applying different layers on bridge decks, which all have their individual function. Typically the work process starts with bringing up the primer on the supporting concrete or steel structure to construct a proper basis for the next layer and to protect the supporting structure from corrosion. On top of the primer the sealing layer (bituminous sheeting) is applied by flame treatment. Consecutive layers are the protection layer (commonly mastic asphalt) and the wearing course (mastic asphalt / stone mastic asphalt). In order to obtain proper quality of construction - affected mainly by the bonding between the individual layers - work process is limited to suitable weather conditions. Hence, mounting often takes place under specific housing to avoid interferences due to weather conditions.

Common damages on bridge decks are blisters that can occur right after the mounting but also after a longer period of time (months or even years). Gerlach (1985), Michalski (1992, 1997) and Rogosch (1994) examined the physical background and developed theories of the origin of blister. According to them, blister evolves from small voids within or between the layers as a result of changing temperatures and pressure conditions of the air-steam mixture in the voids. These pressure induced strains may cause a slow growth of the blister until cracks in the wearing course initiate.

Main causes for small voids are e.g. staining of the grounding (oil, water), missing bonding of the asphalt sheeting to the base or excessive sanding of the primer, which hinders the asphalt sheeting from sealing the voids. Summing up, inaccurate construction is the basis for the origin of blisters.

Michalski (1997) distinguishes two models of blister:

- (i) Blister occurs on dry groundings, when the void is connected to capillaries in the concrete supporting structure. While the temperature increases the mastic asphalt's viscosity decreases and the binder spreads out in the voids, so that its volume is reduced. As a consequence, the inner pressure of the blister rises as long as the amount of exhausting air does not balance the pressure condition due to the flow resistance of the narrow capillaries. This state is characterized as the "phase of overpressure". Decreasing temperatures lead to rising viscosity of the mastic asphalt and declining pressure inside the blister. Air exhausts into the blister as a result of a higher external pressure. The blister shrinks till the inner pressure equals the external pressure. This condition is defined as the "phase of under pressure".

- (ii) Blister occurs on wet groundings, when water rises in the capillary of the concrete base due to their small diameters and causes increasing pressure inside the void. This overpressure is balanced by the capillary pressure and the rising volume of the blister. The water in the capillary is not displaced but the curvature of the meniscus adds up.

Apart from these theories, Wolff et al. (2007) showed that osmotic processes in the contact zone of concrete base and primer, as described above, are not responsible alone for blistering due to their permeability to air and fluids. Therefore, it is not possible that osmotic pressure occurs.

\subsection{Physical basics of thermography}

In the following an introduction of the physical basics of thermography is given in order to point out its ability and advantages related to non destructive testing (Schuster \& Kolobrodov 2004, Maladague 2001). 
Infrared radiation was first discovered by the German astronomer Wilhelm Herschel in 1800. He moved a thermometer through refraction light and realized the maximum temperature in the invisible infrared light spectrum. In 1833, Macedonio Melloni developed the first thermal elements, which allowed changing infrared radiation into an electric signal. In 1920, quantum detectors enabled the measurement of infrared radiation without conversion of the radiation into a temperature, but convert a photon in an energy quantum. Military development led to quantum detector arrays which allow to measure infrared radiation (and thus surface temperatures) with high thermal resolutions without optical scanning.

The basic principle which allows determining the surface temperature from infrared radiation is the fact, that every object has a characteristic radiation, based on the movement of molecules. The intensity of movement depends on the object's temperature and causes electromagnetic radiation with a wavelength between $0.78 \mu \mathrm{m}$ and $20 \mu \mathrm{m}$ (infrared spectrum). In order to convert the intensity of radiation into a temperature, radiation laws must be applied.

The radiating body is a blackbody radiator. A black body at temperature T emits exactly the same wavelengths and intensities which would be present in an environment at equilibrium at temperature $T$, and which would be absorbed by the body. The temperature of the object is directly related to the wavelengths of the light that it emits. The primary law is the Planck Radiation Law, which determines the intensity of radiation emitted by unit surface area into a fixed direction from the blackbody as a function of wavelength for a fixed temperature:

$$
E(\lambda, T)=\frac{2 h c^{2}}{\lambda^{5}} \frac{1}{e^{h c / \lambda k T}-1}
$$

where $\mathrm{h}=$ Planck constant; $\mathrm{k}=$ Boltzmann constant; $\mathrm{c}=$ speed of light; $\lambda=$ wavelength; $\mathrm{T}=$ temperature.

The Planck Law gives a distribution that peaks at a certain wavelength; the peak shifts to shorter wavelengths for higher temperatures (figure 1).

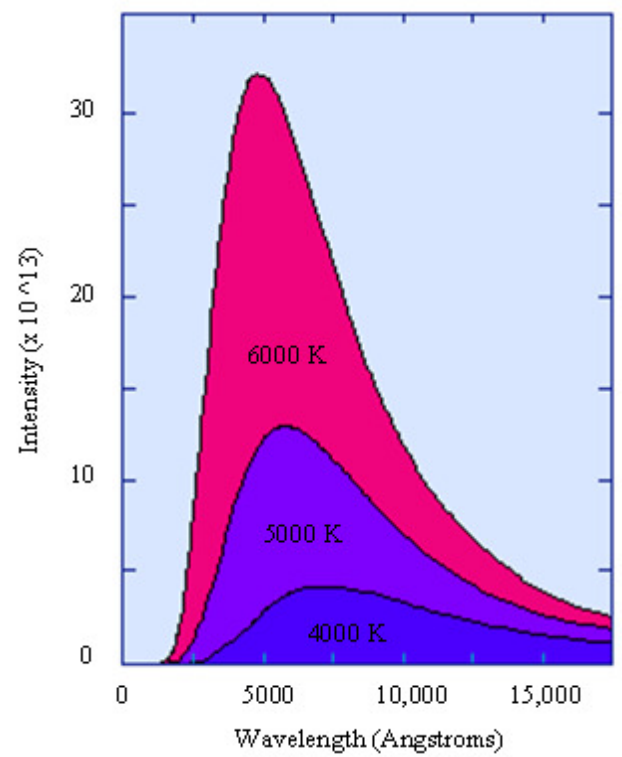

Fig. 1. Distribution of wavelength and intensity of radiation according to Plancks Law

Expressed by the Planck Law, all heated objects emit a characteristic spectrum of electromagnetic radiation, and this spectrum is concentrated in higher wavelengths for cooler bodies. Two other useful radiation laws can be derived from Planck's Law. The integration of (1) gives the total energy emitted at all wavelengths (area under the curves in figure 1) and is known as Stefan-Bolzmann Law:

$$
E=\sigma \cdot T^{4}
$$

where $\sigma=5,67 * 10 \mathrm{erg}-\mathrm{cm}^{2}-\mathrm{K}-\mathrm{sec}$ (Stefan-Bolzmann constant); $\mathrm{T}=$ temperature.

Differentiation of (1) leads to the Wien Displacement Law, which gives the wavelength $\left(\lambda_{\max }\right)$ of the peak of the radiation distribution: 


$$
\lambda_{\max }=\frac{b}{T}
$$

where $\lambda_{\max }=$ peak wavelength; $\mathrm{b}=$ Wien`s displacement constant, equal to $2,897,768.551 \mathrm{~nm} \cdot \mathrm{K}$; $\mathrm{T}=$ absolute temperature of the black body.

For the measurement of surface temperatures by means of thermography, the value of integral between the wavelengths of 8-14 $\mu \mathrm{m}$ is important to obtain a characteristic curve for the temperature and the intensity of radiation. This curve is needed for calibration of a thermographic system.

For measurements of real bodies in practice, there are no perfect emitters that fit the properties of a blackbody as described above. The amount of energy radiated from an object depends on its temperature and its emissivity. Part of the energy hitting an object's surface is absorbed whereas the rest is emitted. The emissivity or emission coefficient $(\varepsilon)$ is the ratio of the energy which a real body radiates and of the energy which a black body emits under the equal temperature. Real bodies always emit less energy than a blackbody, thus the value for $\varepsilon$ ranges between 0 and 1 . The Kirchhoff law describes the relationship between the emissivity $(\varepsilon)$ and the reflectivity $(R)$ :

$$
1-\varepsilon=R
$$

Hence a highly reflective material is a poor emitter of infrared energy and has therefore a low emissivity value.

\subsection{Scope of the study}

Failures in the bonding between the individual layers on bridge decks due to inaccurate construction can finally lead to damages in the wearing course in form of cracks, which enables the infiltration of water causing further damages. Current test methods for "quality assurance" are either destructive to the pavement (e.g. drilling cores) or their application is selective and subjective (e.g. visual examination, tapping surface with hammer). To avoid expensive pavement reconstructions, a test method for a reliable, non destructive quality assurance between construction phases is needed.

Typical damages on bridge decks occur in form of blisters. Due to voids and failures of the bonding in or between the individual layers the thermal transfer to the base is partly disabled. That leads to increasing temperatures at the damaged areas. A common thermographic system allows determining the surface temperature of the pavement without any direct contact. Besides, it is a low cost, easy to use and an area-wide method, which can be applied in realtime. To detect damages by means of thermography, experience in interpreting the thermographic picture is needed, to distinguish between temperature differentials caused by damages (lake of bonding / blister) or differences determined by other influencing factors (e.g. surface shading, thermal material characteristics, adjustments of thermographic system).

\section{Experimental work}

\subsection{Thermographic test equipment}

The portable thermographic system VarioCAM@ hr for long wave infrared spectral range of 7.5 to $14 \mu \mathrm{m}$ was used for damage analyses. The lens images the object scene onto an uncooled microbolometer Focal Plane Array detector at a resolution of $384 \times 288$ pixels. Temperatures can be measured in a range of -40 to $1200^{\circ} \mathrm{C}$ with a temperature resolution of $0.1 \mathrm{~K}$ and measurement accuracy of $1.5 \mathrm{~K}(0 \ldots 100)^{\circ} \mathrm{C}$. A standard lense with a focal length of $25 \mathrm{~mm}$, a minimum focus of $0.3 \mathrm{~m}$ and an Instantaneous Field of View (IFOV) of $1.4 \mathrm{mrad}$ was used.

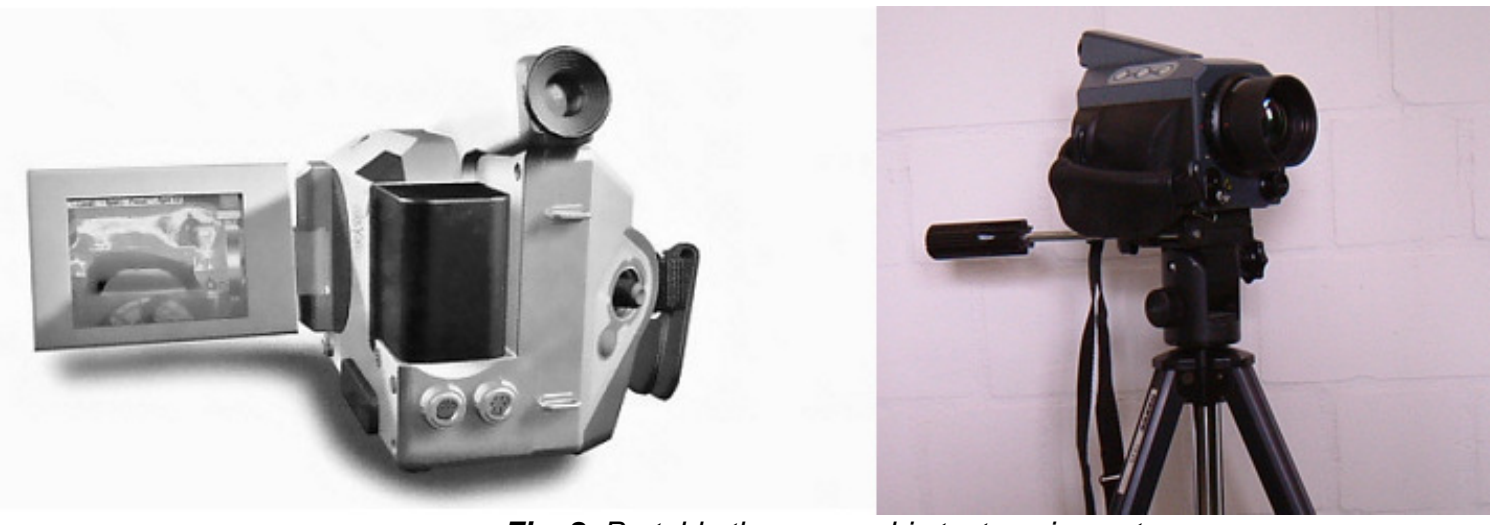

Fig. 2. Portable thermographic test equipment 


\subsection{Results of thermographic examinations}

If blisters reach a certain size, it is possible to detect the damaged spots on the surface by a bulge or, due to traffic loading, caved in blisters in connection with initial cracks. Figure 3 shows a damage spot which was detected by visual examination. As the IR-image of the same spot clarifies, the area of the damaged spot (red coloured) shows higher temperatures on the surface compared to the surrounding area. The difference is about 3 Kelvin and the dimensions of the damaged spot are clearly visible.

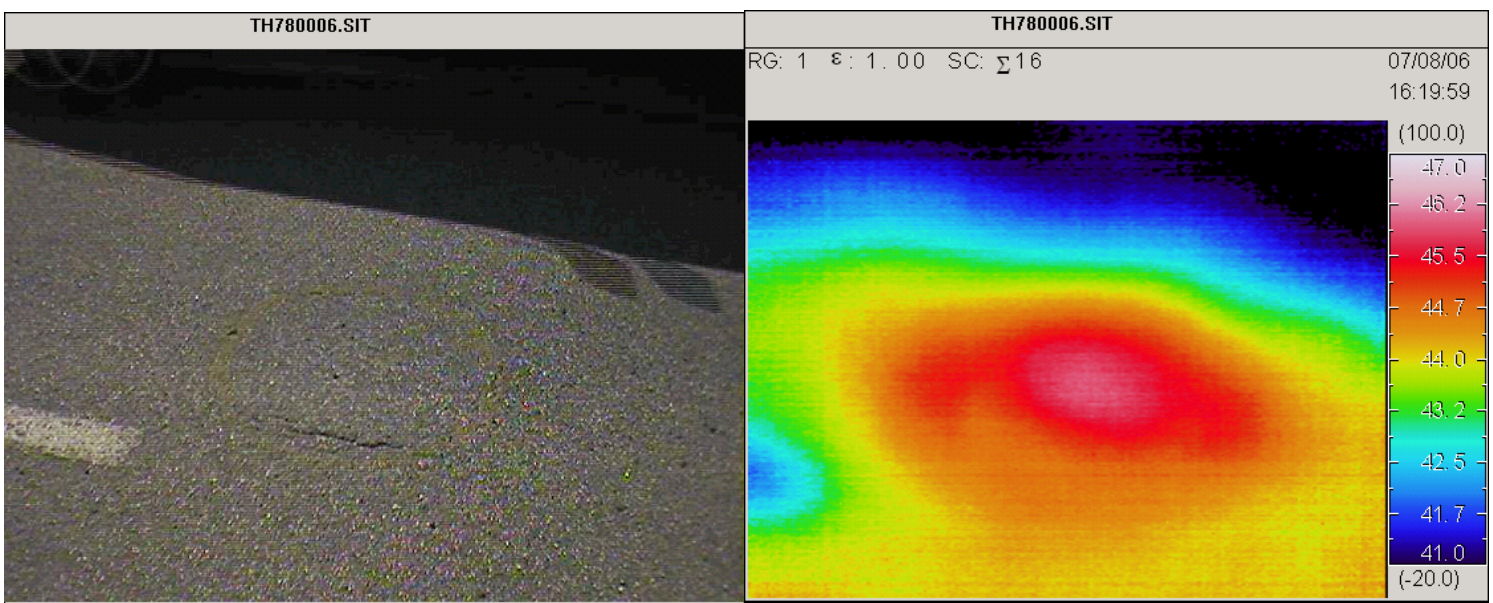

Fig. 3. Blister, on the pavement's surface (left), detected by the IR-image (right).

In order to verify the damage, the spot was opened and bonding between asphalt-sheeting and wearing course was checked (figure 4). The pavement was sawn under dry conditions. The asphalt-sheeting showed wet spots underneath. As demonstrated in the figure by the ruler, the bonding between the concrete base and the asphalt-sheeting is missing completely. Regarding the colour and structure of the sheeting it was concluded that it has not been flamed properly before. The dimensions of the damaged spot fit well with the observations by the IR-image.
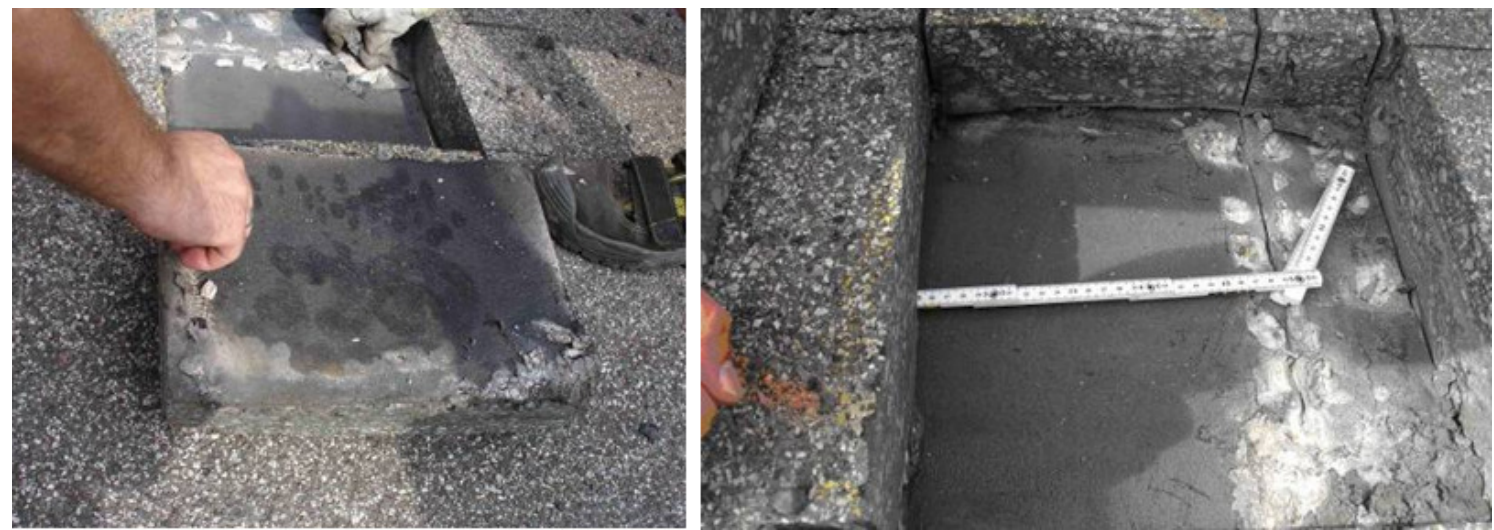

Fig. 4. Opened damaged spot, detected by IR-image before (figure 1); lack of bonding demonstrated by means of a ruler.

Figure 5 illustrates an example of an asphalt-sheeting on a deck without any damage on the surface. Visual examination leads to the assumption of accurate bonding. However, IR-image indicates a lack of bonding. The blister shows a higher surface temperature.

Figure 6 shows the same type of damage. The IR-image indicates a lack of bonding in the middle of the left asphalt-sheeting, running diagonally to the weld. The weld by itself shows a higher surface temperature due to its darker color and higher thermal capacity. On the one hand, this example points out that the thermal capacity of the individual building materials have to be considered when areas of higher surface temperatures are assessed. On the other hand, lack of bonding can be clearly visualized by means of thermography. 


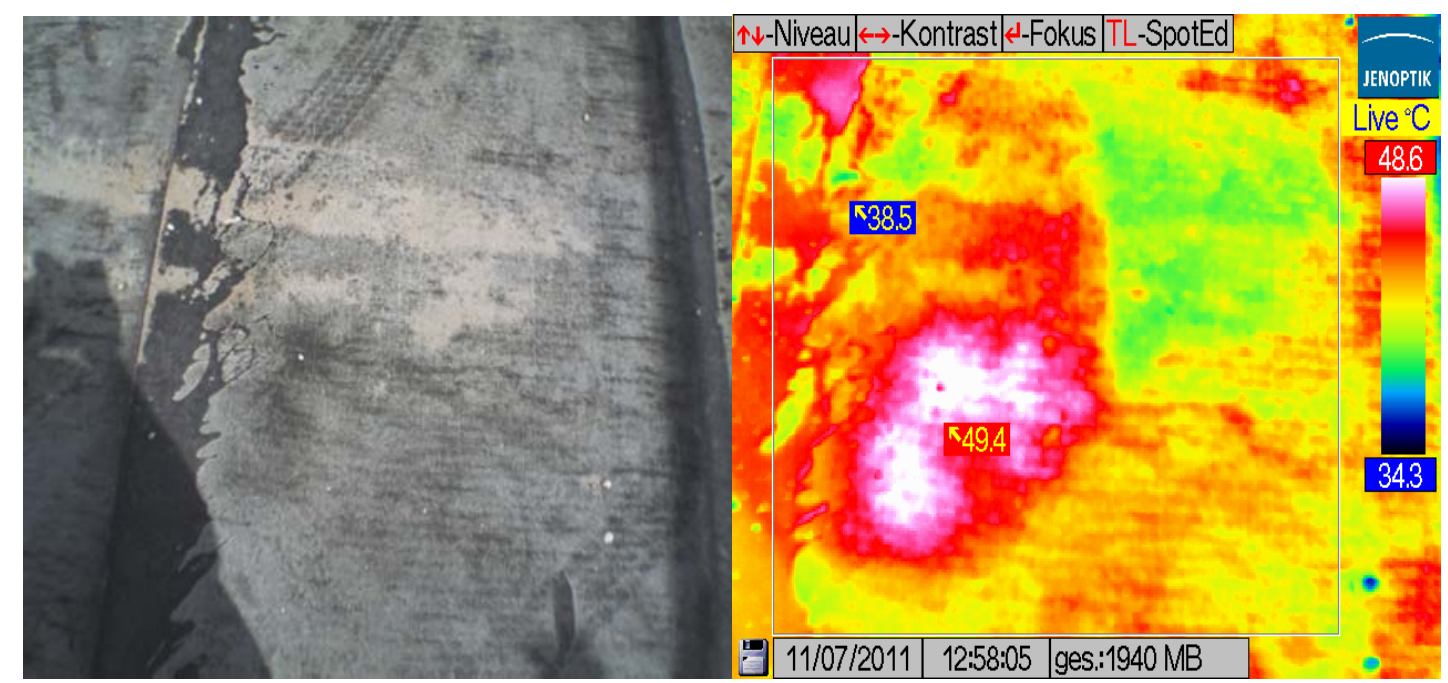

Fig. 5. Blister, on the pavement's surface (left), detected by the IR-image (right).
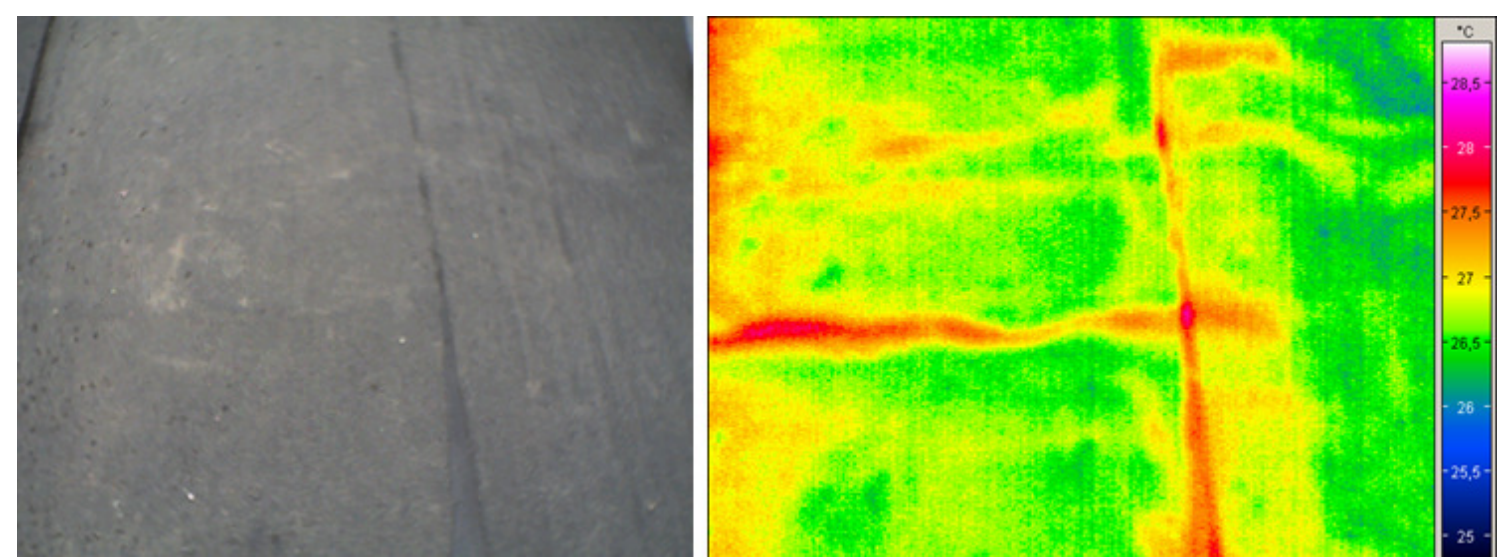

Fig. 6. IR-image shows missing bonding between asphalt-sheeting and base; no damage on surface detectable.

\subsection{Influencing factors on image interpretation}

In order to measure the surface temperature precisely the emission coefficient $\varepsilon$ needs to be adjusted to the material characteristics. In figure 7 , the influence of $\varepsilon$ on the surface temperature is presented.

At first sight, the crash barrier and the concrete verge in the upper part show higher temperatures (up to three degrees) compared to the pavement. As a result of lighter shading, the crash barrier and verge have a higher reflectivity and therefore a lower emissivity than the pavement. For determining the precise surface temperature the emission coefficient of that area needs to be adjusted to e.g. 0.8 compared to 1.0 for the pavement. As the crash barrier is of no interest for assessing the bridge decks quality, $\varepsilon$ was set to 0.85 only for the area marked as "R11" (road marking). The effect of the adjustment is negligible, as the surface temperature of the road marking is increased by only $0.3^{\circ} \mathrm{C}$. Even without any adjustment of $\varepsilon$, a lack of bonding underneath the road marking would be noticeable by a temperature difference. 


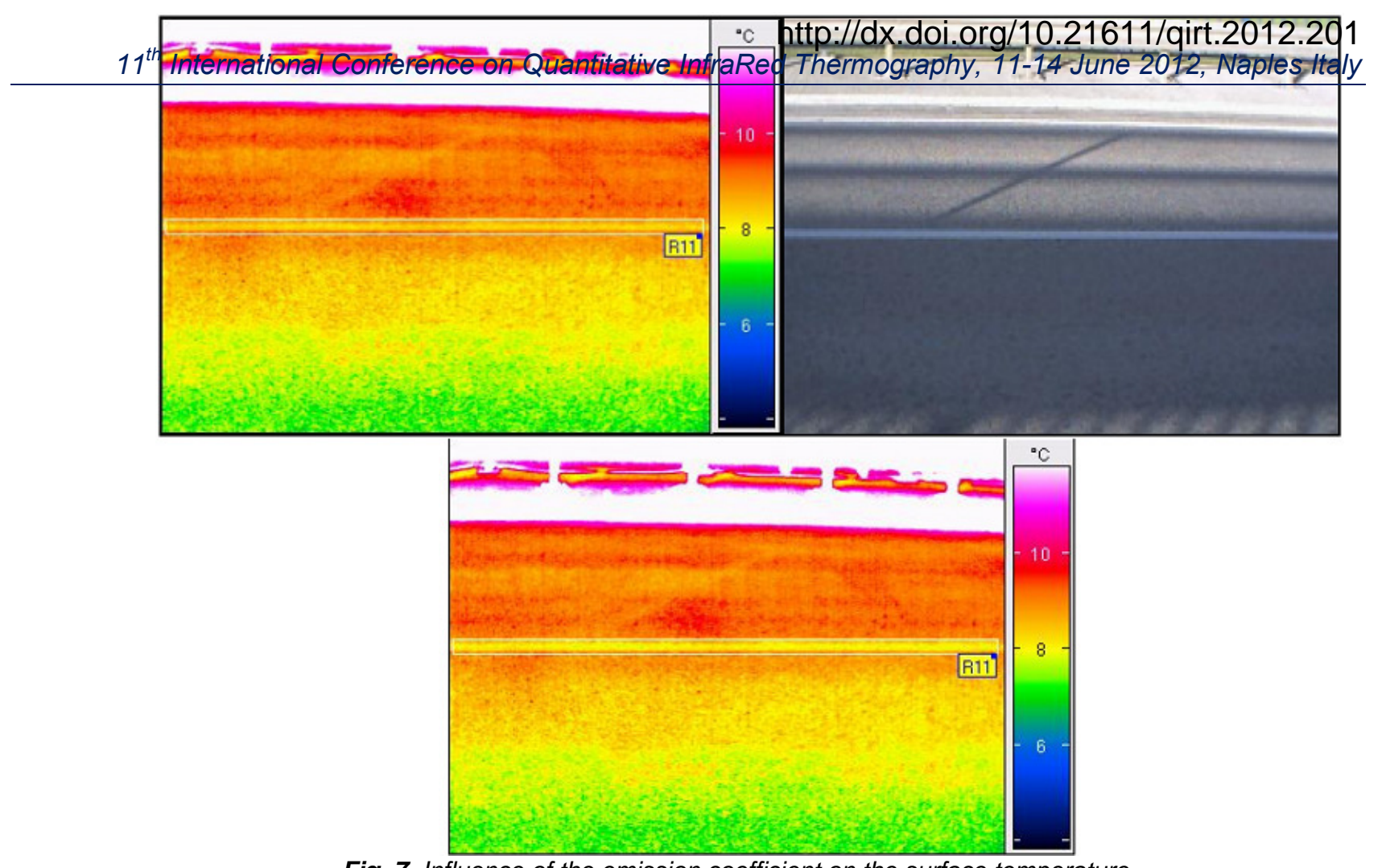

Fig. 7. Influence of the emission coefficient on the surface temperature.

Another effect on the surface temperature can be seen in figure 8 . The front area seems warmer than the back, which is due to the different angle of the camera position.

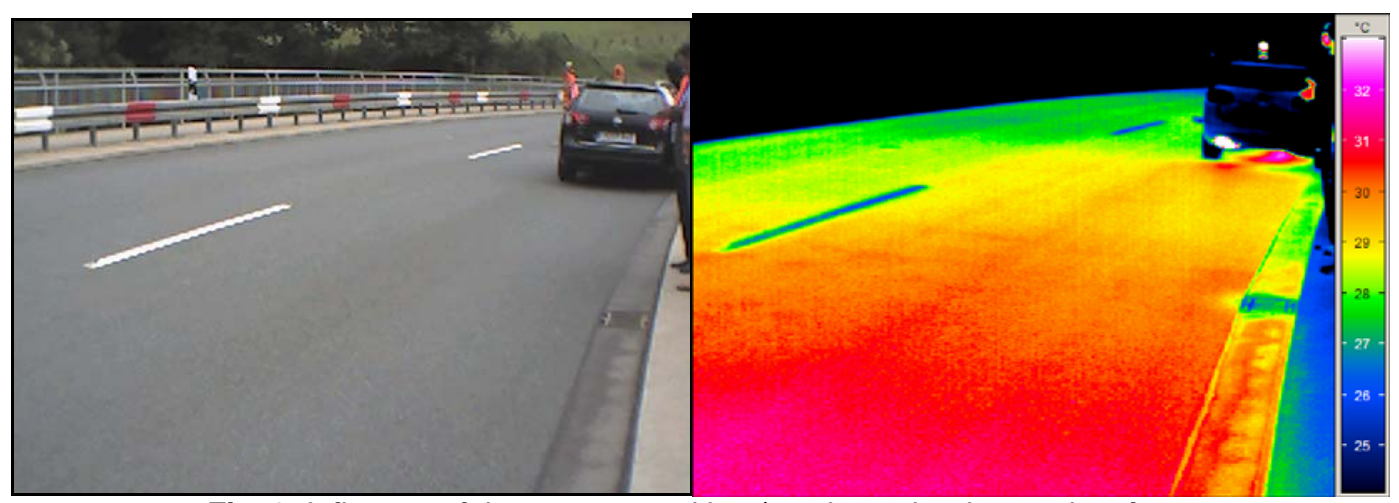

Fig. 8. Influence of the camera position / angle on the detected surface temperature.

Depending on the camera position, the surface temperature of a defined area (yellow) can vary up to $1.3^{\circ} \mathrm{C}$ as tests have shown (Schwarz 2008) (figure 8). It is therefore recommended, to align the camera orthogonal to the surface. The influence of varying angles is thereby eliminated.

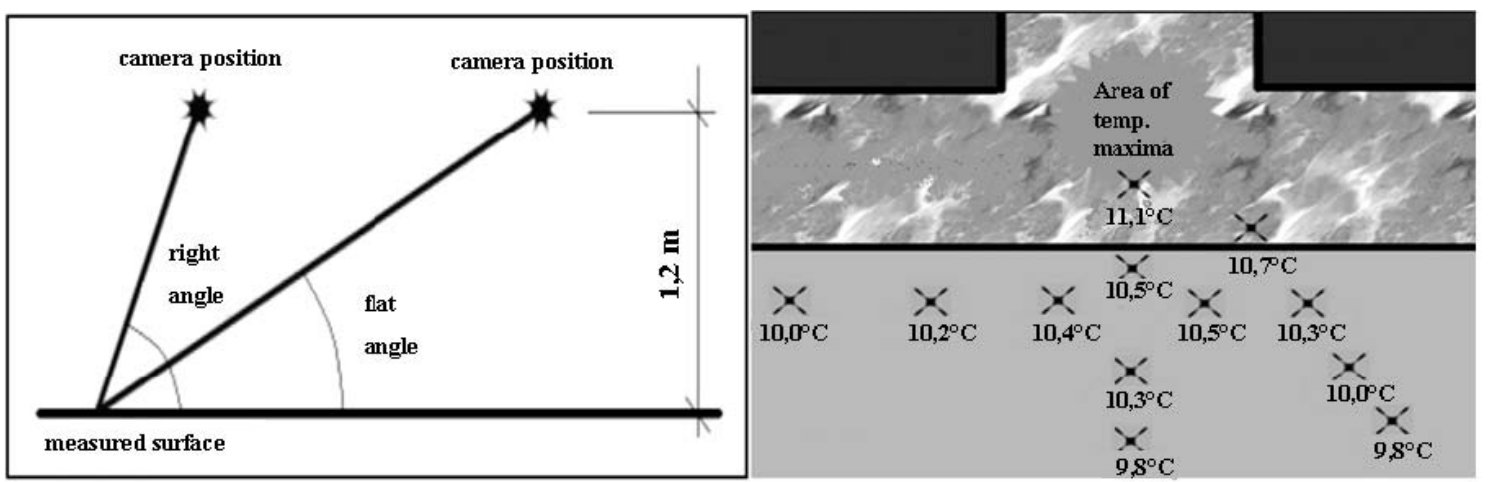

Fig. 9. Influence of the camera position / angle on the detected surface temperature (Schwarz 2008). 
Figure 10 indicates the effect of overlapping of various asphalt sheetings. Visual inspection shows a proper surface, while thermography shows regular attendance of parallel bands with higher surface temperature. They refer to overlapping area between asphalt sheetings under asphalt overlay. This can be explained by the different thermal capacity of the overlapping area with dark bitumen compared to the rest of the asphalt sheeting.
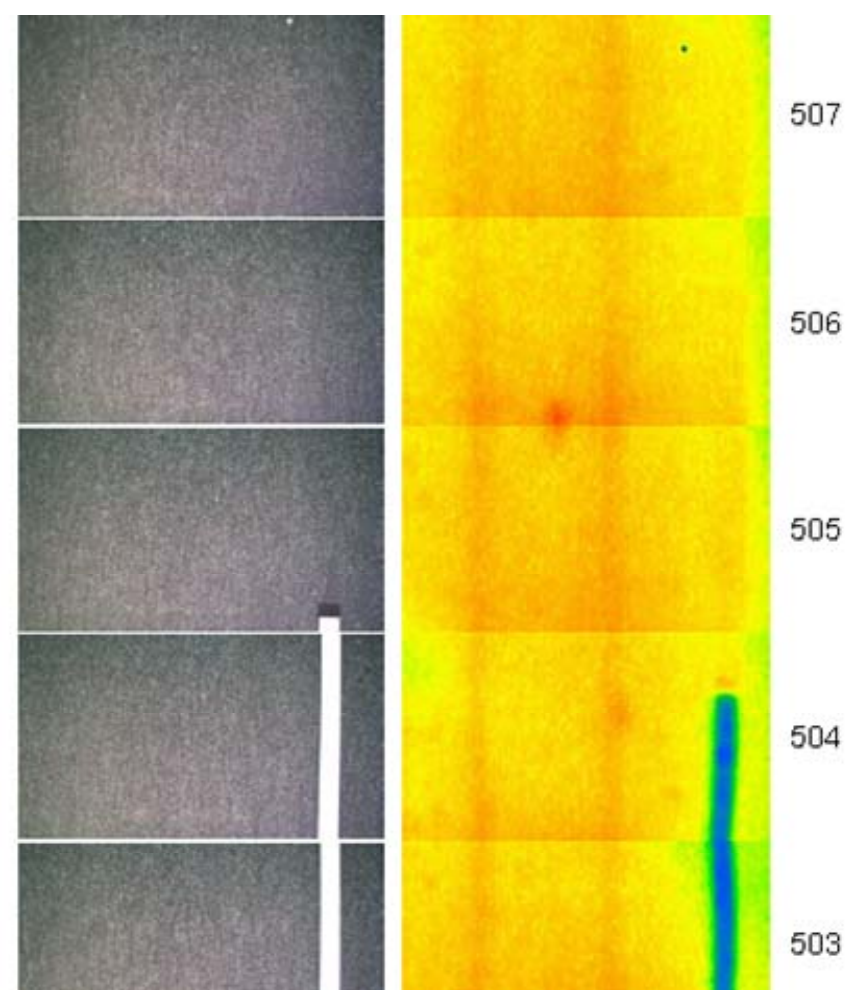

Fig. 10. Thermal effect of overlapping asphalt sheetings.

Inhomogeneities can be selected on the detected surface temperature and on local narrowness, see figure 11. Higher surfaced temperatures (red colour in figure 11) at about $3 \mathrm{~K}$ indicate the position of inhomogeneities.

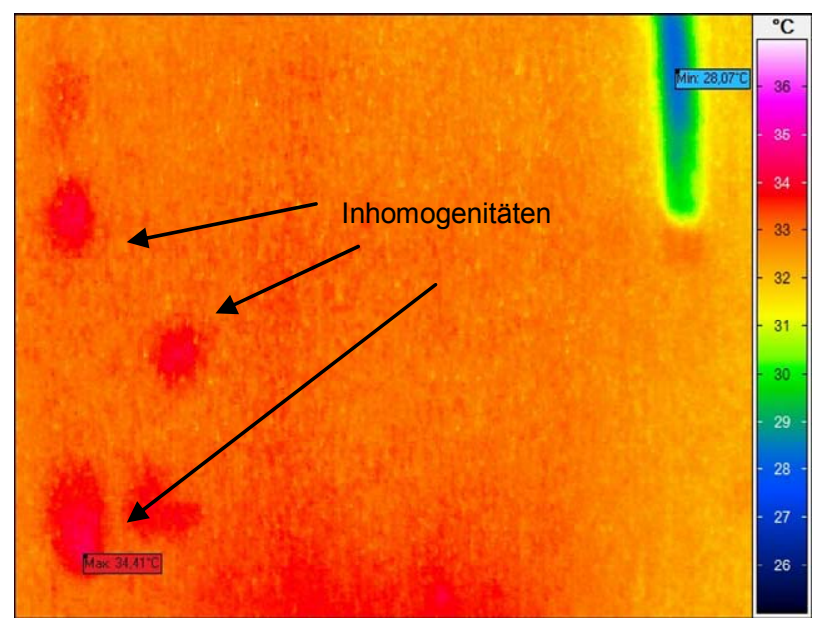

Fig. 11. Local narrowness of inhomogeneities.

More influences on the measured temperature regarding the measurement correction parameters of the thermographic system were investigated by Schwarz (2008). It was concluded, that besides the adjustment of the emission coefficient, none of the varied parameters, such as environmental temperature, transmissivity of the optical channel, or the measure distance had an important impact on surface temperature measurements. 


\section{Concluding remarks}

\subsection{Conlusions}

First examinations of bridge decks by means of thermography give promising results. It is possible to locate damages (blisters, failure in bonding) by temperature differentials on the surface, even before these damages are detected visually. Thermography allows for non destructive quality assurance which is easy to use and cost-efficient. However the interpretation of temperature differentials can be more difficult because influencing factors, as presented in this article, must be taken into account. Although only temperature differentials are decisive for the detection of damages, the surface temperatures should be measured precisely by adapting the emission coefficient to the material's thermal properties. If the proper emission coefficient is unknown, it can be determined experimentally by adjusting $\varepsilon$ until IR temperatures fit the temperatures determined by a contact thermometer. A blackened part of the surface $(\varepsilon=1)$ can be used for the determination alternatively.

\subsection{Perspectives}

First tests showed the basic ability to detect damages on bridge decks by means of thermography. However, further examinations regarding the influence of different pavement surfaces, due to varying rock colours, sanding, staining or different service times on the surface temperature are needed. In addition, the importance of weather conditions on the measurements should be investigated systematically to obtain experience which conditions are most beneficial for detecting temperature differentials. Experience will be summarized in instruction sheets which guarantee that the test procedure is carried out identically under similar conditions. If the thermographic system is fixed to a car, a cadastral-like monitoring of the development of damages, regarding growth, number and position of blister will be possible. The application of the thermograhic examination during the mounting of the layers is preferable but has not been tested in detail yet. It is expected that the temperatures (up to $180^{\circ} \mathrm{C}$ ) make it difficult to detect temperature differentials due to inaccurate bonding during mounting.

\section{Remark}

The presented results are based on IGF-project 16592N/1 sponsored by Arbeitsgemeinschaft industrieller Forschungsvereinigungen „Otto von Guericke" e.V. (AiF) and Bundesministerium für Wirtschaft und Technologie in order to the resolution from Deutscher Bundestag.

\section{REFERENCES}

[1] Gerlach, A. 1985. Zu den Ursachen von Blasenbildungen in bituminösen Fahrbahnbefestigungen Asphaltstraße 19 (7), 341-345.

[2] Maldague, X. P. V. 2001. Theory and Practice of Infrared Technology for Nondestructive Testing. New York: J. Wiley \& Sons.

[3] Michalski, Chr. 1997. Wachsende Blasen in Schichten aus Asphalt unter besonderer Berücksichtigung von Brückenbelägen -Teil 2: Wachsende Blasen in der Praxis. Straße und Autobahn 48 (7), 371-376.

[4] Michalski, Chr. 1997. Wachsende Blasen in Schichten aus Asphalt unter besonderer Berücksichtigung von Brückenbelägen -Teil 1: Entstehen wachsender Blasen. Straße und Autobahn 48 (5), 251-257.

[5] Michalski, Chr. 1992. Modellvorstellungen zur Deutung des Blasenwachstums im Gussasphalt und anderen thermoviskosen Stoffen für den Straßenbau. Mitteilungen aus dem Fachgebiet Konstruktiver Straßenbau im Institut für Verkehrswirtschaft, Straßenwesen und Städtebau der Universität Hannover, (14).

[6] Rogosch, N. 1994. Zur Blasenbildung in thermoviskosen Stoffen unter Berücksichtigung rheologischer Materialkennwerte und geometrischer Randbedingungen. Mitteilungen aus dem Fachgebiet Konstruktiver Straßenbau im Institut für Verkehrswirtschaft, Straßenwesen und Städtebau der Universität Hannover (16).

[7] Schuster, N. \& Kolobrodov, V. G. 2004. Infrarotthermographie. Berlin: Wiley-Vch.

[8] Schwarz, M. 2008. Einsatz der Infrarot-Thermografie zur Beurteilung der erreichten Qualität von Gussasphaltbelägen auf Brücken. Master Thesis. Institut für Straßenwesen, TU Braunschweig.

[9] Wolff, L.; Raupach, M.; Hailu, K. 2007. Ursachen der Blasenbildung bei Reaktionsharzbeschichtungen auf Beton - Welche Rolle spielt die Osmose? Beton-und Stahlbeton 102 (7): 439-449. 